\title{
EDITORIAL
}

\section{Measure, so you can manage}

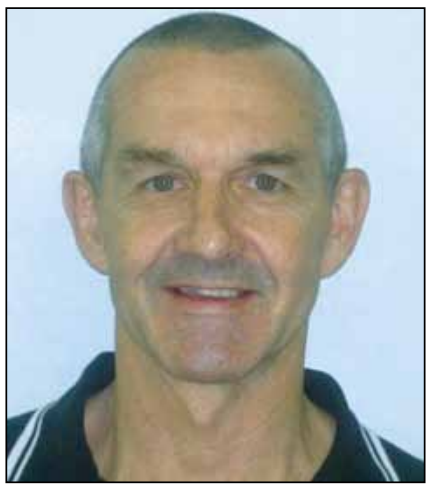

The axiom 'you cannot manage what you don't measure' has been applied in business for several years. Although the origins of the phrase are not clear, it has been adopted as a general management principle in many different spheres of society. In the context of business this statement stresses the importance of collecting data about profits, staff productivity and consumer trends to ensure that the business adapts with the changing demands and is managed in an efficient way. Collecting information regularly enables decisions to be made which are aligned to the changing landscape. These decisions are based on evidence and have a greater probability of being correct compared with decisions based on intuition. Without this ability to forecast it would be very difficult to manage the company in a profitable direction. We see the same application of this principle in medical insurance companies, which employ actuaries to analyse their clients' claim records in an attempt to predict trends and maximise profit. Indeed, this type of analysis has resulted in some of the more proactive companies promoting physical activity as a means of reducing medical costs. Similar examples of management strategies being based on measurement can be found in all types of business.

The principle of 'you cannot manage what you don't measure' can also be applied to sports medicine and exercise science. In fact, this approach should be assumed to be part of a best practice approach of managing patients and sports participants. There are many examples supporting this view, ranging from measuring muscle function during rehabilitation after surgery to managing fatigue in high-performance athletes. While the concept of measurement is easy to understand, the application and interpretation of a measurement is less well understood.

The difficulties of applying and interpreting measurement were highlighted in an excellent paper titled 'Test validation in sport physiology: lessons learned from clinimetrics'. ${ }^{1}$ The authors discuss the field of clinimetrics which focuses on the quality of clinical measurements. This process defines a useful measurement as being reliable, valid and responsive to intervention. Furthermore, the implications of changes in the measurement should be fully understood. In other words how many units of change are necessary for the change in the measurement to be regarded as clinically relevant or meaningful? Data are available, for example, showing that a $21 \%$ gain in the one-repetition-maximum squat exercise is associated with a $2.3 \%$ improvement in sprint performance. ${ }^{2}$ Therefore, while it may be shown in the laboratory that a $5 \%$ increase in squat performance is statistically significant, the relevance of this with regards to sprinting speed is trivial. To reach a conclusion about the merits of the change in the measurement, the measurement error needs to be quantified. Measurement error is the variation that occurs if the measurement were conducted on repeated occasions. For the measurement to be useful the measurement error (also referred to as 'noise') needs to be considerably less that the factor that is being measured (the 'signal'). The precision of the measurement gets reduced the closer the 'noise' is to the smallest worthwhile 'signal'. There is no doubt that the discipline of sports medicine and exercise science needs to be more vigilant about applying the principles of clinimetrics. Without this basic knowledge management of the athlete may be misguided, money will be spent inappropriately and the athletes will bear the brunt of this by failing to reach their potential.

To move the discipline forward all health professionals and scientists who work with athletes need to make a concerted effort to apply the principles of clinimetrics. Firstly, only tests which have undergone evaluation for the determination of their reliability, validity and measurement error should be used. This applies to tests used in clinical rehabilitation through to performance testing for high-performance athletes. Secondly, the minimum worthwhile measurement for each test should be known. This is important for determining whether a change in the measurement is relevant or not. Thirdly, the extent to which a change in measurement in a test translates into the variable that it is designed to measure should also be known. Fourthly, a simple approach can be applied to the changes in the measurement to decide whether the change in the measurement is beneficial, trivial, harmful, or unclear. ${ }^{3}$ The logic behind this approach should be discussed with the coaches and medical support staff so that everyone is aligned in the interpretation. This will make the management of the measurement much easier for everyone involved. Failing to fulfill these 4 points will result in an inability to use the measurements constructively to improve the quality of the management.

This edition of the journal coincides with the 14th Biennial South African Sports Medicine Association conference. The quality and diversity of the abstracts confirm that sports medicine and exercise science is in a healthy state in South Africa!

\section{Mike Lambert}

Editor-in-Chief

1. Impellizzeri FM, Marcora SM. Test validation in sport physiology: lessons learned from clinimetrics. Int J Sports Physiol Perform 2009;4(2):269-277.

2. Young WB. Transfer of strength and power to training to sports performance. Int J Sports Physiol Perform 2006;1(2):74-83.

3. Hopkins WG. How to interpret changes in an athletic performance test. Sportscience 2004;8:1-7. 